\title{
Flow Quantification from 2D Phase Contrast MRI in Renal Arteries using Clustering
}

\author{
Frank G. Zöllner ${ }^{1,2}$, Jan Ankar Monnsen ${ }^{1}$, Arvid Lundervold ${ }^{2}$, Jarle Rørvik ${ }^{1}$ \\ ${ }^{1}$ Department for Radiology, University of Bergen, 5021 Bergen, Norway, \\ ${ }^{2}$ Department for Physiology, University of Bergen, 5009 Bergen, Norway \\ frank.zoellner@biomed.uib.no
}

\begin{abstract}
We present an approach based on clustering to segment renal arteries from 2D PC Cine MR images to measure blood velocity and flow. Such information are important in grading renal artery stenosis and support the decision on surgical interventions like percutan transluminal angioplasty. Results show that the renal arteries could be extracted automatically and the corresponding velocity profiles could be calculated. Furthermore, the clustering could detect possible phase wrap effects automatically as well as differences in the blood flow patterns within the vessel.
\end{abstract}

\section{Introduction}

Renal diseases, caused by e.g. diabetes mellitus or hypertension, can lead to kidney failure that requires renal replacement therapy, where early detection and treatment can delay or prevent this progression towards end-stage renal disease (ESRD). ESDR has an annual mortality of nearly $25 \%$, and in the US the incidence rate of ESRD is 10 new cases per hour. World-wide an accelerating number of people will in the near future suffer from ESRD, with dialysis or kidney transplantation as the costly therapeutic alternatives. Renal artery stenosis (RAS) is
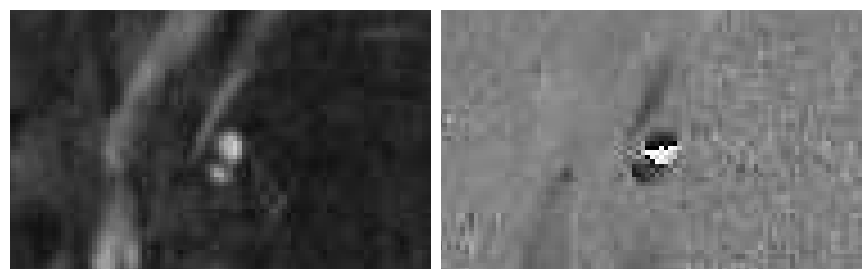

Fig. 1. Images from 2D Cine PC-MRI examination of right renal artery. Left: magnitude image with bright disc shaped region depicting the renal artery, right: corresponding phase image depicting blood flow by grayscale level (midgray level = zero velocity). Dark pixels represent higher blood flow towards the right kidney. The white region next to the dark one depicts a phase wrap effect 
the leading cause of secondary hypertension caused by the reduced flow triggering the auto-regulation (renin-angiotesin) of the systemic circulation including the contralateral kidney. By time this may lead to loss of renal parenchyma in the stenosed kidney and microangiopathy in the glomeruli of the non-stenosed contralateral kidney. To be successful, a percutan transluminal angioplasty (PTA) should be performed before these changes have developed past certain limits in flow-reduction, flow velocity, kidney-size and the overall- and split-renal function. Ignoring these measures may lead to a wrong selection of patients treated with PTA and might be a reason why improvement after PTA has shown to be rather low [1]. Thereby, MRI provides a good tool for measuring these processes [2]. Cine Phase-Contrast MR (PC-MRI) blood flow measurements can be used for non-invasive quantification of renal artery blood flow [3]. During acquisition two images are generated, a magnitude image and a phase image (Fig. 1), encoding blood velocity and direction. From these images the blood flow within the vessel can be quantified and abnormalities in the flow or velocity profiles over the cardiac cycle can be assessed and RAS can be graded.

\section{State of the Art and New Contribution}

Blood flow quantification from PC-MRI acquisition is usually performed by manual or semi-automated delineations of the vessel area $[3,4,5,6]$. This is time consuming and subject to operator dependent variability. Kozerke et al. [7] proposed an active contour approach for vessel segmentation from PC-MRI. A segmentation approach taking the waveform of the velocities into account and applying correlation and thresholding has been proposed in [8]. In this work we present an approach utilising clustering of time series rather than sequential processing of the images finding the vessel lumen frame-by-frame. By this, a segmentation is obtained by grouping flow patterns that are similar during the cardiac cycle. Furthermore, clustering may detect phase wraps caused by too low velocity encoding (VENC), which might mislead an active contour based segmentation. Also, possible differences in the flow pattern within the lumen, e.g. due to partial stenosis, might be detected. In contrast to the segmentation approaches reported in $[7,8]$, the complete flow pattern over the cardiac cycle is taken into account. In addition, manual interactions and observer variability are substantially reduced.

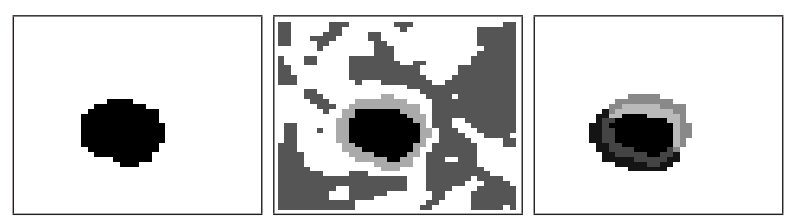

Fig. 2. Clustering results for different $K$. Left to right $K=2, K=4$, and $K=12$ (irrelevant background classes removed for clarity) 


\section{Methods}

Five subjects (3 healthy volunteers, 2 patients) underwent renal blood flow measurements on a $1.5 \mathrm{~T}$ scanner. For the acquisition we followed the procedure described by Schönberg et al. [3]. A trans-sectional slice through the renal vessel is set ca. $1-2 \mathrm{~cm}$ distal of the stenosis for measuring the velocities. We used a ECG gated $2 \mathrm{D}$ cine $\mathrm{PC}-\mathrm{MR}$ sequence with $\mathrm{TE}=37 \mathrm{~ms}, \mathrm{TR}=4 \mathrm{~ms}$, flip angle $=30^{\circ}$, $\mathrm{VENC}=100 \mathrm{~cm} / \mathrm{s}$. Within the cardiac cycle between 20 and 25 images with matrix 256x192 and spatial resolution $0.9 \times 0.9 \mathrm{~mm}^{2}$ and slice thickness $6 \mathrm{~mm}$ were acquired. The blood velocity is measured by (two) so called flow encoding gradients. Their phase difference $(\Delta \phi)$ is then proportional to the velocity $(\nu)$ :

$$
\nu=\left(\frac{\Delta \phi}{\pi}\right) \mathrm{VENC} \quad-\mathrm{VENC}<\nu<\mathrm{VENC}
$$

$\mathrm{VENC}$ is an operator defined parameter for each examination and describes the maximum velocity that can be measured. For the flow analysis, the sequence of phase images were transformed into a 2D matrix of size \# pixels $\times$ time points and then clustered using the K-Means algorithm [9]. We tested three different distance measures (standard euclidean, cosine, and correlation distance) to see which describes the feature space best. Furthermore, we selected several possible numbers of clusters $(K=2, \ldots, 15)$ and evaluated the clustering results by using the DB index [10]. Initialisation of the clustering was by random choice of initial cluster centroids and the clustering was repeated ten times. To increase processing speed the original images were cropped to a small rectangular ROI enclosing the vessel.

\section{Results}

In all our five subjects clustering could discriminate the renal artery from the surrounding tissue and the corresponding velocity and flow profiles could be assessed. A separation of the renal artery from the background could be realised with choosing only $\mathrm{K}=2$ clusters. The analysis of the optimal number of clusters

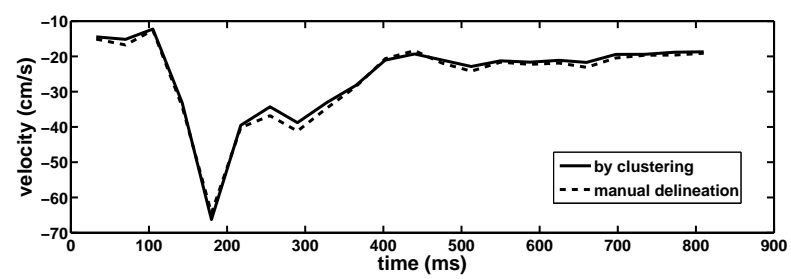

Fig. 3. Calculated mean velocity profiles from manual delineations and from clustering. Data taken from feeding renal artery of the right kidney in a healthy volunteer. Negative velocity values are due to blood flow direction opposite to the read direction in the $2 \mathrm{D}$ $\mathrm{PC}-\mathrm{MR}$ sequence 
[10] revealed that usually two local minima (apart from $\mathrm{K}=2$ ) in the plot of the DB indexes could be observed. This occurred for a small number of clusters (3-5) and a higher one (8-10) depending on the subject and kidney. By selecting such number of clusters, the cluster representing the renal artery was divided in two or more sub-clusters (Fig. 2). Results using the cosine distance and the euclidean metric differed only slightly whereas for the correlation distance, larger regions were segmented.

In one of the data sets, phase wraps occurred (Fig. 1). This could be detected by the clustering approach independently of the number of clusters and distance metric being used (Fig. 4).

Superimposing the resulting clusters onto the original image visually confirms the segmentation results. Also, the calculated velocity profiles in both healthy volunteers and patients have a time course which are typical for those reported in the literature [2]. To better assess our method, we also outlined the vessel lumen manually and calculated the velocity profiles from our datasets. Figure 3 depicts one profile calculated from a manual delineation and results from the clustering. As seen, the two curves show high degree of similarity.

\section{Discussion}

We have presented an approach to semi-automated extraction of velocity and blood flow information from 2D Cine PC-MR images. This was achieved by using K-means clustering to segment the time series of phase images. Our results show that the images are segmented such that the calculated velocity profiles are similar to those obtained after manual delineation - frame-by-frame - of the vessel.

A segmentation of the images into vessel and background could be obtained simply by selecting $K=2$ classes. Selecting a larger number of clusters could discriminate between intra-arterial regions with different flow patterns (Fig. 2 \& $5)$. By this approach we could also detect aliasing (phase wrap) automatically as well as blood flow patterns in the renal artery which might not be easily detected when applying whole vessel segmentation only. In addition, manual interaction
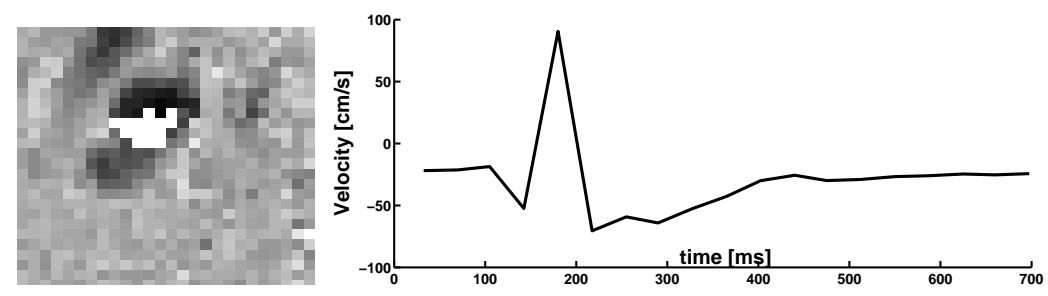

Fig. 4. Clustering result depicting phase wrap in the data. Left: white region represents the phase wrap cluster that is overlayed onto the phase image. Right: calculated velocity profile. At $\mathrm{t}=190 \mathrm{~ms}$ phase wrap occurs. Data is recorded in a healthy volunteer 
Fig. 5. Clusters and corresponding velocity profiles. Left and right: cluster regions (white) overlayed on phase images. Middle: corresponding calculated velocity profiles. The solid line corresponds to the left cluster, the dotted line to the right cluster
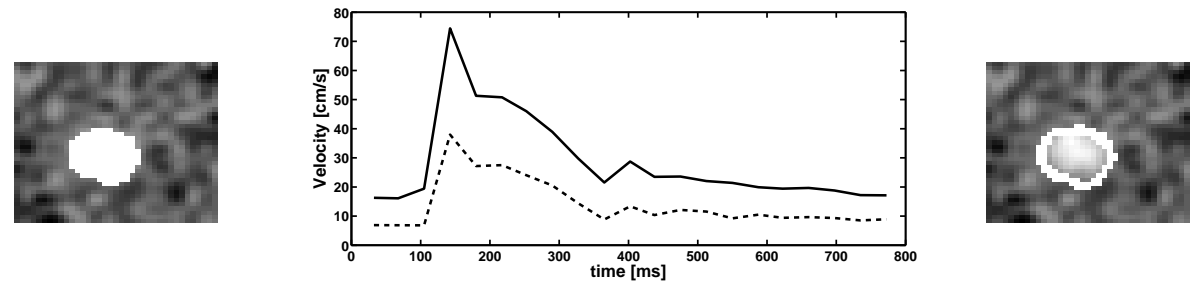

like delineation of the vessel lumen which are mainly performed in clinical practice, are reduced to a simple outlining of a rectangular area containing the vessel in a single frame. In addition, an appropriate number of the clusters has to be selected. We conclude that this approach could be a very helpful tool for clinical assessment and grading of RAS in patients with renal impairment.

\section{References}

1. Krijnen P, van Jaarsveld BC, Deinum J, et al. Which patients with hypertension and atherosclerotic renal artery stenosis benefit from immediate intervention? J Hum Hypertens. 2004;18(2):91-6.

2. Michaely HJ, Schoenberg SO, Oesingmann N, et al. Renal artery stenosis: Functional assessment with dynamic MR perfusion measurements-feasibility study. Radiology. 2006;238(2):586-96.

3. Schoenberg SO, Knopp MV, Bock M, et al. Renal artery stenosis: grading of hemodynamic changes with cine phase-contrast MR blood flow measurements. Radiology. 1997;203(1):45-53.

4. Graves MJ, Brewin MP, Priest AN, et al. Automated analysis of cine phase contrast velocity images using scored guided erosion. Proc ISMRM. 2001;9:835.

5. Bax L, Bakker CJG, Klein WM, et al. Renal blood flow measurements with use of phase-contrast magnetic resonance imaging: normal values and reproducibility. J Vasc Interv Radiol. 2005;16.

6. Florez YN, Moratal D, Forner J, et al. Semiautomatic analysis of phase contrast magnetic resonance imaging of cerebrospinal fluid flow through the aqueduct of Sylvius. Magma. 2006;19(2):78-87.

7. Kozerke S, Botnar R, Oyre S, et al. Automatic vessel segmentation using active contours in cine phase contrast flow measurements. J Magn Reson Imaging. 1999;10(1):41-51.

8. Alperin N, Lee SH. PUBS: pulsatility-based segmentation of lumens conducting non-steady flow. Magn Reson Med. 2003;49(5):934-44.

9. MacQueen JB. Some methods for classification and analysis of multivariate observations. Proc Berkeley Symposium on Mathematical Statistics and Probability. 1967;1:281-97.

10. Davies DL, Bouldin DW. A cluster separation measure. IEEE Trans Pattern Anal Mach Intell. 1979;1(2):224-7. 